\title{
A Review on the Role of Chymase in Hypertensive Heart
}

\section{Disease.}

\section{Bright Boafo Boamah ${ }^{1 *}$ Edward Kwaku Armah ${ }^{2}$, \&Gifty Oppong Boakye ${ }^{3}$}

${ }^{\mathbf{1}}$ Department of Pharmacology, Kwame Nkrumah University of Science and Technology, Kumasi, Ghana.

${ }^{2}$ Department of Chemical Engineering, Durban University of Technology, Durban, South Africa.

${ }^{3}$ Department of Mechanical Engineering, University of Leeds, Leeds, United Kingdom

\begin{abstract}
Hypertensive heart disease is the commonest cardiac complication resulting from longstanding hypertension. Hypertensive patients inevitably develop hypertensive heart disease but the intensity and progression vary. Poor control is associated with a fast progression and worse outcome while the outcome of adequately controlled blood pressure is antithetical to the former. Various biochemical mediators have been implicated in hypertension and its related complications. Angiotensin-II is involved in both hypertension and remodeling of the cardiac and vascular architecture. The focus on Chymase and its inhibitors in modulating angiotensin-II levels have cast some doubts on the efficacy of solely using angiotensin converting enzyme inhibitors.
\end{abstract}

Keywords: Chymase, Hypertensive heart disease, Angiotensin-II, Blood pressure, Inhibitors 


\section{INTRODUCTION}

Hypertensive heart disease (HHD) is a sequela to hypertension which inevitably develops especially when blood pressure is poorly controlled [1]. Its development is influenced by interplay of genetic and non-genetic factors [2]. Poorly controlled hypertension has been implicated as the main reason for development of left ventricular hypertrophy (LVH) [3], however, the role of biochemical mediators such as angiotensin-II, endothelin-I, norepinephrine and urotensin-II cannot be overlooked [4]. Angiotensin-II plays a key role in homeostasis by regulating arterial tone and fluid balance [5], but becomes toxic to tissues in pathological states [6]. Angiotensin-II is the main biochemical mediator involved in LVH [7], although its inhibitors do not reduce blood pressure significantly compared to calcium channel blockers, diuretics and vasodilators [8]. This stresses on the impact of biochemical mediators causing left ventricular hypertrophy independent of the level of blood pressure.

Previously, angiotensin-II activity on tissues were known to be as a result of circulating angiotensin-II [ 9 ] but multiple studies have shown the presence of locally produced angiotensin-II through enzymatic pathways [10]. Angiotensin-II is found in both tissues and plasma [11], with its effect varying and depending on the site and duration of action [12]. Angiotensin converting enzyme inhibitors were discovered as a result of the evidence pointing towards angiotensin-II in pathogenesis of vascular and cardiac hypertrophy [7]. Discovery of multiple antihypertensive drugs have curtailed most deleterious effects of hypertension but much requires to be done as emerging evidence prove some pitfalls in treatment strategies [13].

A significant number of patients, 20 to $40 \%$ are unresponsive to angiotensin converting enzyme inhibitors [14]. A myriad of clinical trials has confirmed the greater antihypertensive effect on combining angiotensin converting enzyme inhibitor and angiotensin-II type-I receptor blocker as opposed to using angiotensin converting enzymes inhibitors solely [15]. This further bolsters the presence of an Angiotensin Converging Enzymes (ACE)-independent pathway in angiotensin-II formation. In the last two decades, the activity of alternate pathways mediating the conversion of angiotensin-I to angiotensin-II were discovered [16], challenging renin-angiotensin-aldosterone system as the sole pathway. The raveling of an alternate pathway was discovered by Cornish and colleagues in 1978, who reported the existence of a possible alternate pathway in angiotensin-II formation in animal models [12]. A study by Urata et al, 1990 confirmed the presence of an ACE-independent pathway in the formation of angiotensin-II in human heart [17]. Although several serine proteases such as trypsin, cathep$\sin \mathrm{G}$, Chymase and chymotrypsin were involved in in-vitro conversion of angiotensin-I to angiotensin-II, cathepsin G and Chymase were found to be of essence to human cardiovascular system [17]. In a study by Takai, human vascular tissue angiotensin-II formation was partially blocked withAngiotensin Converging Enzymes Inhibitors (ACEIs)[18]. This signifies the essence of non-ACE dependent angiotensin-II formation in humans. Most of the angiotensin-II was inhibited by Chymase inhibitor, chymostatin [19]. This explains the phenomenon of 'angiotensin escape' where angiotensin-II returned to pretreatment levels in patients with angiotensin converting enzyme inhibitors. Angiotensin-II mediates inflammatory actions and may induce Chymase activity leading to a vicious cycle as more angiotensin-II is released by the process [20]. Circulating levels of angiotensin-II is dependent on angiotensin 
converting enzymes while tissue angiotensin-II is dependent mainly on Chymase [21]. The presence of serum protease inhibitors limits the activity of Chymase in circulation [22]. However, serum Chymase in hypertensive patients is high compared to non-hypertensive patients [23]. The elevation in serum Chymase signifies the role of Chymase in hypertension which is supposed to remain undetected or at low levels due to the presence of circulating serine protease inhibitors. This review however seeks to address the role of Chymase in hypertensive heart diseases.

\section{DISTRIBUTION AND ACTIVITY OF CHYMASE IN CARDIAC TISSUE}

The activity of angiotensin converting enzymes play a crucial role in formation of angiotensin-II but the role of Chymase in pathophysiology of hypertension and hypertensive heart disease needs to be recognized [24]. In the physiological state, Chymase activity is currently not clearly established but in pathological states like hypertension, cardiac failure, atherosclerosis and myocardial infarction, Chymase is induced [19]. Angiotensin-II levels in the cardiac tissue are derived from both local sources and from the circulating pool in serum [25]. Local cardiac Chymase cleaves about $80 \%$ of angiotensin-I to angiotensin-II as opposed to angiotensin converting enzymes [26]. The distribution of Chymase and angiotensin converting enzymes were first determined by Urata and colleagues, who examined the distribution of these enzymes in patients with heart failure.

Angiotensin converting enzymes are abundant in the right ventricle of about 3-fold compared to the left ventricle similarly, the right atrium had a concentration of about 2-fold higher than left atrium [17]. This depicts the crucial role of Chymase enzyme in the left ventricular hypertrophy as well as differential response of cardiac chambers to ACEI therapy. Chymase granules are predominantly located in the interstitium as well as the myocardium and might be responsible for extracellular matrix angiotensin-II formation which leads to excessive collagen deposition and fibrosis [27]. In cardiac tissue, Chymase is known to induce the expression of transforming growth factor-beta (TGF-b) which mediates myocardial fibrosis [28] aside angiotensin-II. Mast cells are ubiquitous in human tissues but the concentration of Chymase is tissue specific, some tissues like buccal mast cells have no or little Chymase [29]. In a study by Nussberger et al, Chymase was immune to serum serine proteinase when bound to heparin sulphate [30]. This might explain the reason why Chymase remains active although serine inhibitors exist in the extracellular matrix. Systolic heart failure is one of the sequelae to remodeling induced by angiotensin-II [31].

Ruthie and colleagues in 2008 discovered a strong correlation between non-ischemic systolic heart failure and heart Chymase activity. The deleterious effect of unopposed Chymase activity was associated with poor clinical outcome [32]. In a pre-clinical study, Chymase was able to induce hypoxia in myocardium leading to remodeling of cardiac architecture independent of blood pressure [33] although this has not been firmly established in humans. Acute myocardial infarction resulting from sudden occlusion of the coronary artery is dependent on sudden rupture of the atheroma cap. Metalloproteinase- 9 produced by Chymase is implicated in the rupture [34]. However, an initial study by Holubarsch et al did not find any inotropic effect with angiotensin-II from Chymase in the heart [35]. This suggests that possibly, angi- 
otensin-II produced by Chymase is of different molecular structure and might have only remodeling effects on the heart and vascular tissues.

\section{DISTRIBUTION AND ACTIVITY OF CHYMASE IN VASCULAR TISSUES}

The vascular physiology oscillates between two main states of dilation or constriction based on the sympathetic tone. Vasoconstriction is the main vascular state in hypertension and its related disorders [36]. Angiotensin-II mediates vasoconstriction within the acute phase of exposure in hypertension [37], while chronicity eventually results in hypertrophy and intimal anatomical aberrations [38]. Vascular angiotensin-II is derived from circulating angiotensin-II produced by ACE [39], while the local angiotensin-II in the vessel is produced by both the ACE and Chymase [40]. Chymase activity is significant in the blood vessels following vascular injury [41]. A study on the impact of Chymase and ACE on angiotensin-II formation in the blood vessels revealed almost $70 \%$ production by the former enzyme. Captopril was infused into vascular tissues and partial vasoconstriction was achieved signifying the role of a possible alternate pathway to angiotensin-II formation [42].

In a similar study by Takai and colleagues, 2001, he found almost $90 \%$ of angiotensinII in vessels produced by Chymase [43]. In a study of activity of ACE and Chymase in coronary arteries among patients with coronary heart disease and heart failure, it was found out that a blockade of either enzymes resulted in partial inhibition if angiotensin-II [44]. This shows that a complete blockade of angiotensin-II will require more than just an ACEI. Aneurysms have also been implicated in the activity of Chymase through the angiotensin-II mediated matrix metalloproteinase (MMP)-9 activation [45]. Activation of MMP-9 has also been observed in acute myocardial infarction from rupture of the atherosclerotic capsule triggering thrombosis and hence coronary occlusion [46].

Cardiac dysfunction post-myocardial infarction is mitigated by the use of Chymase inhibitors. Of note, Chymase inhibitors although reduces angiotensin-II have no impact on reducing blood pressure compared to angiotensin converting inhibitors [47]. Probably, because it has no direct impact on kinins and renin angiotensin aldosterone system. Thus, Chymase can be used in normotensive patients for its vascular and cardiac anti-remodeling benefits without causing hypotension.

\section{CLINICAL IMPLICATIONS}

The burden of hypertensive heart disease remains a serious problem within the medical practice [49]. Influence of co-morbid conditions on prognosis of HHD cannot be understated [50]. However, the discovery of novel drugs led to the control of hypertension and a halt in the progression of its complications [51]. In hypertensive heart disease, angiotensin-II blockade has been the main target for the reversal of various phenotypic expressions such as left ventricular hypertrophy, ischemic heart disease, heart failure, arrhythmia and valvular heart disease [52]. The induction of left ventricular hypertrophy by hypertension has been found to even occur without elevated blood pressure [53 ], which justified the use of angiotensin modulating drugs (ACEIs and ARBs) even in low renin and salt sensitive populations like blacks [ 54 ]. 
Unfortunately, the morbidity and mortality with hypertensive heart disease still remains high especially in Black populations although antihypertrophic agents such as angiotensin modulating drugs are employed [55]. Could there be a variation in antihypertrophic response to angiotensin-II modulating drugs in blacks? Existence of various co-morbidities such as dyslipidemia, chronic kidney disease and diabetes mellitus worsen HHD [ 56]. Angiotensin converting enzyme inhibitors serve as the first line drugs among the angiotensin modulating classes in treating hypertensive heart disease [57]. This raises the concern as to whether angiotensin converting enzyme inhibitors alone are able to reverse or halt hypertrophy. Combination of angiotensin-II receptor type-I blockers and angiotensin converting enzyme inhibitors have proved effective in totally blocking both tissue and plasma angiotensin-II [58]. However, the combination of Angiotensin Receptor Blockers (ARBs) and ACEIs is limited by the possible side effects usually at high doses; hyperkalemia, orthostatic hypotension and angioedema which are associated with it $[59,60]$.

The reasoning behind the combination stems from the fact that alternate pathways are now known to be involved in formation. In clinical studies, restenosis after percutaneous coronary intervention is one of the main complications following this procedure. The use of ARBs and ACEIs produced varying results; the former inhibited restenosis significantly while the latter had no major influence [61]. These results galvanize further studies on the role of Chymase inhibitors in such circumstances, since Chymase not only increases angiotensin-II but other remodeling factors like TGF-alpha. Chymase inhibitors in both animal models and human models have shown to play a significant role in angiotensin-II formation especially in the cardiac and vascular tissues [62]. In a study by, chymostatin inhibited more than $90 \%$ of Chymase [43], while alpha-1 antitrypsin inhibited about 95\%[ 63] Reversal of various stages of hypertensive left ventricular hypertrophy was associated with the use of Chymase inhibitors alone compared to angiotensin converting enzyme inhibitors but a combination provided maximum angiotensin-II inhibition [ 64 ]. In Blacks, poor blood pressure control in response to angiotensin modulating drugs in hypertension has been clearly defined [65]. However, its benefits to heart, kidneys and blood vessels are mainly extrapolated from clinical trials with an assumption that bigger numbers will produce same results. Further therapeutic studies on the role of Chymase inhibitor in hypertensive heart disease management should be considered.

Chymase has been shown to play a role in hypertension and its related disorders through angiotensin-II [66]. Chymase has also been recently implicated in patients with diabetic nephropathy resistant to antihypertensive medications [67]. A study by park and colleagues explored one of the possible mechanisms in unresponsiveness in patients with diabetes nephropathy to ACEIs. It was realized that angiotensin-II produced in the kidneys of these patients were predominantly from Chymase and hence angiotensin converting enzyme inhibitors had no major impact on its blockade [68]. Conditions affecting production of serum proteins have been shown to affect the levels of Chymase. In the serum, Chymase activity is limited by the presence of serine protease inhibitors such as alpha- ${ }_{1}$ antitrypsin [34]. The liver is the anabolic site for alpha- ${ }_{1}$ antitrypsin and hence conditions such as liver cirrhosis and hepatoma can reduce its levels significantly [69]. Genetic disorders affecting alpha-1 antitrypsin levels 
(PiSS, PiSZ and PiZZ) are known to cause emphysema [ 70 ] but the role in hypertension has not been fully researched on since studies on Chymase is still embryonic.

Albuminuria is one of the markers of kidney disease [71]; it has been realized to cause a significant loss of alpha- 1 antitrypsin in patients with nephropathies. This loss can also explain the reason why ACEIs are less effective in diabetics with nephropathy. Multiple trials have shown the effectiveness in reducing proteinuria on combining ACEIs and ARBs compared to using either agents alone [72].

\section{CONCLUSION}

Angiotensin converting enzyme inhibitors remain the first line agent in the class of angiotensin modulating drugs in the management of hypertensive heart disorder. Current evidence points towards a possible impact of an alternate pathway, Chymase in angiotensin-II production. Chymase istissue bounded and plays a crucial role in both vascular and cardiac remodeling. Chymase at tissue level produces majority of angiotensin-II compared to angiotensin converting enzyme. It serves as the connection between the immune system and cardiovascular diseases. Inhibition of Chymase activity results in maximum extenuation of angiotensin-II, mainly tissue form which improves patient's outcome in not only hypertensive heart diseases but also in renal and inflammatory conditions. Pharmacodynamics of Chymase inhibitors differ from ACEI, the former, although it causes a significant reduction in angiotensin-II do not have any influence on blood pressure control compared to the latter. A combination of an ACEI and a Chymase inhibitor will yield a maximum reduction in angiotensinII and its related complications compared to angiotensin converting enzyme inhibitors and angiotensin-II type-I receptor blockers. This will obviate the untoward effects from using the latter combination. Further studies required in the use of Chymase inhibitors solely and in combination with angiotensin converting enzyme inhibitors.

\section{DECLARATION OF INTEREST}

The authors declare that this article's content has no conflict of interest.

\section{ACKNOWLEDGEMENT}

The authors are thankful to the Kwame Nkrumah University of Science and Technology, Kumasi for the use of their library facilitiesduring the research findings.

\section{REFERENCES}

[1] Geronikolou, S. A., \&Cokkinos, D. (2016). The Progression of Hypertensive Heart Disease to Left Ventricular Hypertrophy and Heart Failure. Hypertension and Cardiovascular Disease, 59-74.

[2] Pang, H., Han, B., Li, Z., \& Fu, Q. (2015). Identification of molecular markers in patients with hypertensive heart disease accompanied with coronary artery disease. Genetics and Molecular Research, 14(1), 93-100. 
[3] Jie Liu, H. X. (2013). Relationship between Nocturnal Blood Pressure and Left Ventricular Hypertrophy in Hypertensive Patients. Journal of Hypertension- Open Access, 03 (03).

[4] Pavlyukova, E., Bahmetiva, T. and Karpov, R. (2006). Left ventricular longitudinal function in hypertensive patients with left ventricular hypertrophy regression. European Journal of Echocardiography, 7, S108-S108.

[5] Sullivan, J. C. (2015). Sex Differences in Angiotensin II Hypertension. Sodium and Water Homeostasis, 91-117.

[6] Nishiyama, A., Seth, D. M., \&Navar, L. G. (2003). Angiotensin II type 1 receptor-mediated augmentation of renal interstitial fluid angiotensin II in angiotensin II-induced hypertension. Journal of Hypertension, 21(10), 1897-1903.

[7] Abu Arab, T. M., El Nozahi, M., Wahid, W., \& Hanna, H. F. (2017). Angiotensin II as an Indicator of Left ventricular Hypertrophy in Uncontrolled Treated Hypertensive Patients. International Journal of Cardiovascular Research, 06 (02).

[8] Baguet, J. P., Legallicier, B., Auquier, P., \&Robitail, S. (2007). Updated Meta-Analytical Approach to the Efficacy of Antihypertensive Drugs in Reducing Blood Pressure. Clinical Drug Investigation, 27(11), 735-753.

[9] Ardaillou, R. (1999). The relative roles of circulating and tissue renin-angiotensin systems. Nephrology Dialysis Transplantation, 14(2), 283-286.

[10] Lindpaintner, K. (1993). Selective Activation of Cardiac Angiotensinogen Gene Expression in Post-infarction Ventricular Remodeling in the Rat. Journal of Molecular and Cellular Cardiology, 25(2), 133-143.

[11] Takai S, Jin D, Miyazaki M. New approaches to blockade of the renin-angiotensin-aldosterone system: chymase as an important target to prevent organ damage. J Pharmacol Sci.2010;113: 301309.

[12] Cornish KG, Joyner WL, Gilmore JP: Direct evidence for the presence of a different converting enzyme in the hamster cheek pouch. Circ Res 1979;44:540-544.

[13] Antoniou, S., Saxena, M., Hamedi, N., De Cates, C., Moghul, S., Lidder, S., Lobo, M. D. (2015). Management of Hypertensive Patients With Multiple Drug Intolerances: A Single-Center Experience of a Novel Treatment Algorithm. The Journal of Clinical Hypertension, 18 (2), 129-138.

[14] Becker, B.F., All because of the mast cell: blocking the angiotensin receptor-1 should be better than inhibiting ACE (theoretically). Cardiovasc Res.,92(1):7- 9,2011.

[15] Li, M., K. Liu, J. Michalicek, J. A. Angus, J. E. Hunt, L. J. Dell'Italia, M. P. Feneley, R. M.Graham, and A. Husain. 2004. Involvement of chymase- mediated angiotensin II generation in blood pressure regulation. J. Clin. Invest. 114: 112-120.

[16] Uehara Y, Miura S, Yahiro E, Saku K. Non-ACE pathway-induced angiotensin II production.Curr Pharm Des 2013; 19: 3054-9. 
[17] Urata H, Kinoshita A, Misono KS, et al: Identification of a highly specific chymase as the majorangiotensin II- forming enzyme i; the human heart. J Biol Chem 1990;265:22348-22357.

[18] Takai S, Jin D, Miyazaki M. New approaches to blockade of the renin-angiotensin-aldosterone system: chymase as an important target to prevent organ damage. J Pharmacol Sci.2010;113: 301-309.

[19] Doggrell, S. A., \&Wanstall, J. C. (2005). Cardiac chymase: pathophysiological role and therapeutic potential of chymase inhibitors. Canadian Journal of Physiology and Pharmacology, 83(2), 123-130.

[20] Kennedy, S., Wu, J., Wadsworth, R.M., Lawrence, C.E., Maffia, P., Mast cells and vascular diseases. PharmacolTher., 138(1):53-65,2013.

[21] Arakawa,K., Urata ,H., Hypothesis regarding the pathophysiological role of alternative pathways of angiotensin II formation in atherosclerosis. Hypertension, 36(4):

$638-41,2000$.

[22] Miyazaki, M. (2002). Development and Application of Chymase Inhibitors: Preface. The Japanese Journal of Pharmacology, 90(3), 205.

[23] Hristova, M., Penev, M., Stanilova, S., and Miteva, L. (2015). Preliminary study of the serum chymase levels in patients with arterial hypertension. Trakia Journal of Science, 13(2), 160165 .

[24] McDonald, J. E., Padmanabhan, N., Petrie, M. C., Hillier, C., Connell, J. M., andMcMurray, J. J. (2001). Vasoconstrictor Effect of the Angiotensin-Converting Enzyme-Resistant, ChymaseSpecific Substrate [Pro11D-Ala12] Angiotensin I in Human Dorsal Hand Veins: In Vivo Demonstration of Non-ACE Production of Angiotensin II in Humans. Circulation, 104(15), 1805-1808.

[25] Xiao, H. D., Fuchs, S., Frenzel, K., Teng, L., \& Bernstein, K. E. (2004). Circulating versus Local Angiotensin II in Blood Pressure Control: Lessons from Tissue-Specific Expression of Angiotensin-Converting Enzyme (ACE). Critical Reviews in Eukaryotic Gene Expression,14(1-2), 137-146.

[26] Chester, A., \& Borland, J. (2000). Chymase-dependent angiotensin II formation in human blood vessels. Journal of Human Hypertension, 14(6), 373-376.

[27] Nakajima, M., and Naya, N. (2002). Development and Application of Chymase Inhibitors: Development of a Chymase Inhibitor: Pharmacological Characterization of a Chymase Inhibitor in Inflamed Tissue Remodeling and Fibrosis. The Japanese Journal of Pharmacology, 90(3), 206-209.

[28] Doggrell, S. A., \&Wanstall, J. C. (2005). Cardiac chymase: pathophysiological role and therapeutic potential of chymase inhibitors. Canadian Journal of Physiology and Pharmacology, 83(2), 123-130.

[29] Jones, T. G., \&Gurish, M. F. (2014). Methods for the Study of Mast Cell Recruitment and Accumulation in Different Tissues. Mast Cells, 69-78.

[30] Nussberger, J., D. B. Brunner, B. Waeber, and H. R. Brunner. 1986. Specific measurement of angiotensin metabolites and in-vitro generated angiotensin II in plasma. Hypertension. 8:476482. 
[31] Wang, Y., Seto, S., and Golledge, J. (2012). Angiotensin II, sympathetic nerve activity and chronic heart failure. Heart Failure Reviews, 19(2), 187-198.

[32] Amir, R. E., Amir, O., Paz, H., Sagiv, M., Mor, R., Sagiv, M., \& Lewis, B. S. (2008). Genotypephenotype associations between chymase and angiotensin-converting enzyme gene polymorphisms in chronic systolic heart failure patients. Genetics in Medicine, 10(8), 593598.

[33] Matsumoto C, Hayashi T, Kitada K, Yamashita C, Miyamura M, Mori T, Ukimura A, Ohkita M, Jin D, Takai S, Miyazaki M, Okada Y, Kitaura Y, Matsumura Y. Chymase playsan important role in left ventricular remodeling induced by intermittent hypoxia in mice. Hypertension 2009; 54: 164-71.

[34] Jin D, Takai S, Yamada M, Sakaguchi M, Kamoshita K, Ishida K, et al. Impact of chymase inhibitor on cardiac function and survival after myocardial infarction. Cardiovasc Res. 2003; 60: 413-420.

[35] Holubarsch C, Hasenfuss G, Schmidt-Schweda S, et al. 1993.Angiotensin I and II exert inotropic effects in atria! but not in ventricular human myocardium: an in vitro study under physiological experimental conditions. Circulation;88:1228-1237.

[36] Joyner, M. J. (2006). Too Much Is Not Enough: Hypertension and Sympathetic Vasoconstriction in Contracting Muscles. Hypertension, 48(4), 560-561.

[37] Dinh, Q. N., Drummond, G. R., Sobey, C. G., and Chrissobolis, S. (2014). Roles of Inflammation, Oxidative Stress, and Vascular Dysfunction in Hypertension. BioMed Research International, 2014, 1-11.

[38] Takayanagi, T., Kawai, T., Forrester, S. J., Obama, T., Tsuji, T., Fukuda, Y., Eguchi, S.(2015). Role of Epidermal Growth Factor Receptor and Endoplasmic Reticulum Stress in Vascular Remodeling Induced by Angiotensin IINovelty and Significance. Hypertension, 65(6), 13491355 .

[39] Abu Arab, T. M., El Nozahi, M., Wahid, W., and Hanna, H. F. (2017). Angiotensin II as an Indicator of Left ventricular Hypertrophy in Uncontrolled Treated Hypertensive Patients. International Journal of Cardiovascular Research, 06(02).

[40] Dilsizian, V., Loredo, M., Ferrans, V. J., Jagoda, E. M., Eckelman, W. C., \& Shirani,

J. (2002). Evidence for increased angiotensin II type 1 receptor immunoreactivity in peri-infarct myocardium of human explanted hearts. Journal of the American College of Cardiology, 39, 365 .

[41] Okunishi, H., Oka, Y., Shiota, N., Kawamoto, T., Song, K., \& Miyazaki, M. (1993). Marked Species-Difference in the Vascular Angiotensin II-Forming Pathways: Humans versus Rodents. The Japanese Journal of Pharmacology, 62(2), 207-210.

[42] Husain A, Kinoshita A, Sung SS,: Human heart chymase, and Linduaintner K. The Cardiac Renin-Angiotensin System. Futura Publishing, New York, 1994, pp 309331.

[43] Takai S, Sakaguchi M, Jin D, Yamada M, Kirimura K, Miyazaki M. Different angiotensin IIforming pathways in human and rat vascular tissues. ClinChim Acta. 2001;305:191-195.

[44] Petrie, M. C., Padmanabhan, N., McDonald, J. E., Hillier, C., Connell, J. M., and McMurray, J. J. (2001). Angiotensin converting enzyme (ACE) and non-ACE dependent angiotensin II 
generation in resistance arteries from patients with heart failure and coronary heart disease. Journal of the American College of Cardiology, 37(4), 1056-1061.

[54] Williams, S. F. (2014). African Americans, hypertension and the renin angiotensin system. World Journal of Cardiology, 6(9), 878.

[55] Calvin, R., Winters, K., Wyatt, S. B., Williams, D. R., Henderson, F. C., and Walker, E. R. (2003). Racism and Cardiovascular Disease in African Americans. The American Journal of the Medical Sciences, 325(6), 315-331.

[56] Rushton, C., and Kadam, U. (2014). Impact of non-cardiovascular disease comorbidity on cardiovascular disease symptom severity: A population-based study. International Journal of Cardiology, $175(1), 154-161$.

[57] Flather, M. D., Yusuf, S., Køber, L., Pfeffer, M., Hall, A., Murray, G., Braunwald, E. (2000).Long-term ACE-inhibitor therapy in patients with heart failure or left-ventricular dysfunction:a systematic overview of data from individual patients. The Lancet, 355(9215), 1575-1581.

[58] Morisco, C., and Trimarco, B. (2014). Angiotensin Converting Enzyme Inhibitors and AT1 Antagonists for Treatment of Hypertension. ACEi and ARBS in Hypertension and Heart Failure, $1-39$.

[59] REHMAN, A., ISMAIL, S., NAING, L., ROSHAN, T., \& RAHMAN, A. (2007). Reduction in Arterial Stiffness With Angiotensin II Antagonism and Converting Enzyme Inhibition: A Comparative Study Among Malay Hypertensive Subjects With a Known Genetic Profile. American Journal of Hypertension, 20(2), 184-189.

[60] Cheng, J., Zhang, W., Zhang, X., Han, F., Li, X., He, X., Chen, J. (2014). Effect of AngiotensinConverting Enzyme Inhibitors and Angiotensin II Receptor Blockers on All-Cause Mortality, Cardiovascular Deaths, and Cardiovascular Events in Patients With Diabetes Mellitus. Journal of American Medical Association Internal Medicine, 174(5), 773.

[61] Ruilope LM, Aldigier JC, Ponticelli C, Oddou-Stock P, Botteri F, Mann JF: Safety of the combination of valsartan and benazepril in patients with chronic renal disease. European Group for the Investigation of Valsartan in Chronic Renal Disease. Journal of Hypertension18: 89 -95, 2000.

[62] Mogensen, C. E., Neldam, S., Tikkanen, I., Oren, S., Viskoper, R., Watts, R. W. and Cooper, M. E. (2000). Randomized controlled trial of dual blockade of renin-angiotensin system in patients with hypertension, microalbuminuria, and non-insulin dependent diabetes: the candesartan and lisinopril microalbuminuria (CALM) study. BMJ, 321(7274), 1440-1444.

[63] Takai S, Jin D, Sakaguchi M, et al. A novel chymase inhibitor, 4-[1-([bis-(4-methyl-phenyl)methyl]-carbamoyl) 3-(2-ethoxy-benzyl)-4-oxo-azetidin e-2-yloxy]-benzoic acid (BCEAB),suppressed cardiac fibrosis in cardiomyopathic hamsters. J PharmacolExpTher 2003; 305:17-23.

[64] Takai S, Jin D, Miyazaki M. New approaches to blockade of the renin-angiotensin-aldosterone system: chymase as an important target to prevent organ damage. J Pharmacol Sci. 2010;113: 301309.

[65] Brewster, L. M., and Seedat, Y. K. (2013). Why do hypertensive patients of African ancestry respond better to calcium blockers and diuretics than to ACE inhibitors and $\beta$ adrenergic blockers? A systematic review. BMC Medicine, 11(1). 
[66] Li, M., Liu, K., Michalicek, J., Angus, J. A., Hunt, J. E., Dell'Italia, L. J., Husain, A. (2004). Involvement of chymase-mediated angiotensin II generation in blood pressure regulation. Journal of Clinical Investigation, 114(1), 112-120.

[67] Huang, X. R. (2003). Chymase Is Upregulated in Diabetic Nephropathy: Implications for an Alternative Pathway of Angiotensin II-Mediated Diabetic Renal and Vascular Disease. Journal of the American Society of Nephrology, 14(7), 1738-1747.

[68] Park, S., Bivona, B. J., Ford, S. M., Xu, S., Kobori, H., De Garavilla, L., and Harrison-Bernard, L. M. (2012). Direct Evidence for Intrarenal Chymase-Dependent Angiotensin II Formation on the Diabetic Renal Microvasculature. Hypertension, 61(2), 465-471.

[69] Carrell RW, Lomas DA.( 2002). Alpha 1-antitrypsin deficiency-a model for conformational diseases. New England Journal of Medicine, 346,45-53.

[70] Bowlus, C. L., Willner, I., Zern, M. A., Reuben, A., Chen, P., Holladay, B. and Strange, C. (2005). Factors associated with advanced liver disease in adults with alpha1-antitrypsin deficiency. Clinical Gastroenterology and Hepatology, 3(4), 390-396.

[71] Ahmed, A., Ibrahim, F. H. and Nahas, M. E. (2009). Management of chronic kidney disease: controlling hypertension and reducing proteinuria. Chronic Kidney Disease, 207-230.

[72] Bakris, G. L. (2008). Slowing Nephropathy Progression: Focus on Proteinuria Reduction.Clinical Journal of the American Society of Nephrology, 3(1), S3-S10. 